\begin{tabular}{|c|c|}
\hline Title & Innate immunity and vaccine \\
\hline Author(s) & Seya, Tsukasa \\
\hline Citation & $\begin{array}{l}\text { V accine, 28(50), 8041-8042 } \\
\text { https:/doi.org/10.1016/.vaccine.2010.09.023 }\end{array}$ \\
\hline Issue Date & 2010-11-23 \\
\hline Doc URL & http:/hdl.handle.net/2115/44770 \\
\hline Type & article (author version) \\
\hline File Information & V ac28-50_8041-8042.pdf \\
\hline
\end{tabular}

Instructions for use 


\title{
Innate immunity and vaccine
}

Tsukasa Seya

Department of Microbiology and Immunology, Hokkaido University Graduate School of Medicine, Kita-ku, Kita-15, Nishi-7, Sapporo 060-8638 Japan

Running title: Prologue

Corresponding author: Tsukasa Seya, M.D., Department of Microbiology and Immunology, Hokkaido University Graduate School of Medicine, Kita 15, Nishi 7, Kita-ku, Sapporo 060-8638, Japan. Tel: 8111706 5073, Fax: 8111706 7866,

E-mail: seya-tu@pop.med.hokudai.ac.jp

Keywords: TLR, MyD88, TICAM-1 (TRIF), dendritic cells, NK activation.

\begin{abstract}
Immune adjuvant is an artificial PAMP for potentiating various immune responses. Vaccine represents one event that is capable of inducing immune response caused by antigen and PAMP stimuli, which act on antigen-presenting dendritic cells (mDCs). Here, we introduce the pathways by which CTL and NK cells are driven through mDC maturation in response to adjuvants.
\end{abstract}

Microbial pattern molecules (PAMP, pathogen-associated molecular patterns) are agonists of pattern-recognition receptors, a representative of which is Toll-like receptor. Adjuvants are non-infectious artificial PAMP, typically administered with the target antigen (Ag) in order to enhance the host immune response (1). However, the mechanism by which these reagents enhance immunity had not clearly been understood, until the recent progress on elucidation of the ligand properties of Toll-like receptors (TLRs) and TLR-mediated DC maturation (2). The accumulated evidence on TLR-dependent DC maturation has solidified the current understanding that DC TLRs confer direction of effector driving on the DCs that present antigens. Now, we hold that Ags determine the object toward which immune cells are proliferated whereas adjuvant determines what effectors will be selected for 
immunological output (1). The fundamental concepts of the immune system should be re-evaluated through the understanding of TLR-mediated DC immune responses, which will also revolutionize the concepts related to vaccination.

In myeloid DCs (mDCs), a representative Ag-presenting cells, the two major arms of the innate immune signaling pathway, the MyD88 and TICAM-1 (TRIF) pathways, have been identified through the investigation of TLR signaling (2). TLR3 represents the sensor of dsRNA of viral origin and recruits TICAM-1 (3). TICAM-1 links the type I IFN-inducing pathways in mDCs of both human and mouse $(2,3)$. TLR4 recruits both MyD88 and TICAM-1 (2). TLRs other than TLR3 can take the MyD88 pathway. Hence, the representative inflammatory responses in TLR pattern-recognition are rooted in the properties of the adaptors MyD88 and TICAM-1. In myeloid DCs, these pathways play a significant role in differential maturation.

Using BCG-CWS as the TLR2/4 adjuvant, we found that MyD88 is an adaptor essential for induction of cross-priming in mDCs (4). MyD88 -/- mice have been reported to far less induction of CTL against exogenous Ags and TLR2/4 adjuvants (4). Cytokines and NF- $\kappa \mathrm{B}$-inducing factors may be required for $\mathrm{mDC}$ cross-priming, although the molecular mechanism whereby MyD88 can induce responses related to cross-presentation in mDCs.

We have used polyI:C for evaluating the TICAM-1 (TRIF) potential in mDC maturation (5). The TICAM-1 pathway allows mDCs to activate IRF-1 and IRF-3, which in turn activate the IFN- $\beta$ promoter as well as unidentified NK-driving factors. The data imply that cross-priming and the NK-driving signal are also dependent upon TICAM-1, but the transcription factors utilized by TICAM-1 are wholly distinct from those of MyD88. We found that mDC TICAM-1-mediated NK activation largely relies on the IRF-3-derived NK-activating molecule (INAM) which promotes mDC-NK cell contact (6), in addition to the reported soluble mediators IL-15, IFN- $\alpha$, and IL-12p70. Thus, the mode by which mDCs matured differs in the MyD88 and the TICAM-1 pathways. If an appropriate adjuvant is conjugated with vaccine, NK cells can be activated for eradication of microbes.

We have analyzed how mDCs acquire effector-driving functions by focusing on the innate immune response (1). Live vaccines usually contain microbe-specific Ags and PAMPs. Since DNA per se has an adjuvancy, DNA vaccine also includes PAMPs. Nevertheless, potential vaccines have not been established for some viral infections. For example, HCV, HIV and influenza infections have their own problems. Low titers of antiviral antibody and CTL induction may be dissolved by developing efficient adjuvants 
by inducing appropriate effectors. In this stand, an effective strategy for tackling the issue of low immune response against vaccines has yet to be proposed with obstinacy infectious diseases. Even the fundamental immune aberrance present in the focal nests of infection has not been grasped by researchers in some virus-induced persistent infections.

There is almost no information concerning the molecular mechanisms driving these effector cells in mDCs. Each DC subset seems to correspond to a specific effector, although the selection mechanism by which DCs induce various effectors is not clear in most instances. However, it is known from mouse models that splenic CD8+ DCs induce Treg (7) and NK cells (8) in the mouse spleen, and lamina propria pDCs in the mouse enteric canal promotes IgA production (9). In addition, CD70+/CD11c+ DCs induce Th17 cells by the ATP of enterobacteria (10), and bone-marrow (BM)DCs markedly activate NK cells via the TICAM-1 pathway (11). It is known that pDCs induce tremendous amounts of IFN- $\alpha$ in response to CpG DNA through TLR9. Although what molecular background supports this pDC phenotype has long been unknown, pDC-specific events should regulate the activation of IRF-7 (12, T. Kaisho in this presentation). Further examples of DC subsets that preferentially function with specific effectors will likely be demonstrated through practical experiments.

For future studies, it is necessary to determine the potential of peptide-conjugating materials including Ags and inflammation-inducing reagents. A number of reports have suggested that adjuvants can greatly increase the efficiency rate of treatment, although there are no criteria to fairly evaluate the function of adjuvants in vaccine recipients or patients. The method for stimulating DCs needs to be carefully selected as systemic administration of inflammation-inducing material can also lead to the acceleration or exacerbation of infection at the same time. In this case, the route and molecule that selectively raises the degree of DC maturation without severe malicious inflammation should be clarified. The design of DC maturation can be manipulated without helping flare inflammation. In the future, we hope that through continued research, patients will have access to convenient and highly effective prophylactic immunotherapy.

\section{References}

1. Seya T, Shime H, Ebihara T, Matsumoto M. Pattern recognition receptors of innate immunity and their application to tumor immunotherapy. Cancer Sci. 2010; (in press).

2. Akira S, Uematsu S, Takeuchi O. Pathogen recognition and innate immunity. Cell. 2006; 124: 783-801. 
3. Matumoto M, Seya T. TLR3: interferon induction by double-stranded RNA including poly(I:C). Adv Drug Deliv Rev. 2008; 60: 805-12.

4. Akazawa T, Masuda H, Saeki Y, Matsumoto M, Takeda K, Tsujimura K, Kuzushima K, Takahashi T, Azuma I, Akira S, Toyoshima K, Seya T. Adjuvant-mediated tumor regression and tumor-specific cytotoxic response are impaired in MyD88-deficient mice. Cancer Res. 2004; 64: 757-64.

5. Akazawa T, Ebihara T, Okuno M, Okuda Y, Shingai M, Tsujimura K, Takahashi T, Ikawa M, Okabe M, Inoue N, Okamoto-Tanaka M, Ishizaki H, Miyoshi J, Matsumoto M, Seya T. Antitumor NK activation induced by the Toll-like receptor 3-TICAM-1 (TRIF) pathway in myeloid dendritic cells. Proc Natl Acad Sci U S A. 2007; 104: 252-7.

6. Ebihara T, Azuma M, Oshiumi H, Taniguchi T, Matsumoto M, Seya T. Identification of INAM, a polyI:C-inducible membrane protein, that participates in dendritic cell-mediated natural killer cell activation. J Exp Med. 2010 (in press).

7. Yamazaki S, Dudziak D, Heidkamp GF, Fiorese C, Bonito AJ, Inaba K, Nussenzweig MC, Steinman RM. CD8+ CD205+ splenic dendritic cells are specialized to induce Foxp3+ regulatory T cells. J Immunol. 2008; 181: 6923-33.

8. Miyake T, Kumagai Y, Kato H, Guo Z, Matsushita K, Satoh T, Kawagoe T, Kumar H, Jang MH, Kawai T, Tani T, Takeuchi O, Akira S. Poly I:C-induced activation of NK cells by CD8 alpha+ dendritic cells via the IPS-1 and TRIF-dependent pathways. J Immunol. 2009; 183: 2522-8.

9. Tezuka H, Abe Y, Iwata M, Takeuchi H, Ishikawa H, Matsushita M, Shiohara T, Akira S, Ohteki T. Regulation of IgA production by naturally occurring TNF/iNOS-producing dendritic cells. Nature. 2007; 448: 929-33.

10. Atarashi K, Nishimura J, Shima T, Umesaki Y, Yamamoto M, Onoue M, Yagita H, Ishii N, Evans R, Honda K, Takeda K.. ATP drives lamina propria $\mathrm{T}(\mathrm{H}) 17$ cell differentiation. Nature. 2008; 455: 808-12.

11. Ebihara T, Shingai M, Matsumoto M, Wakita T, Seya T. Hepatitis C virus-infected hepatocytes extrinsically modulate dendritic cell maturation to activate $\mathrm{T}$ cells and natural killer cells. Hepatology. 2008; 48: 48-58.

12. Hoshino K, Sugiyama T, Matsumoto M, Tanaka T, Saito M, Hemmi H, Ohara O, Akira $\mathrm{S}$, Kaisho T. IkappaB kinase-alpha is critical for interferon-alpha production induced by Toll-like receptors 7 and 9. Nature. 2006; 440: 949-53. 